\title{
Influencia de la inflamación crónica prostática en muestras de biopsia sextante en los niveles séricos de PSA total y PSA libre
}

\author{
L. Gómez Pérez, A. Budía Alba, A. Benedicto Redón, F.J. Delgado Oliva, J.L. Palmero Martí, \\ J.F. Jiménez Cruz
}

Servicio de Urología. Hospital Universitario La Fe. Valencia.

Actas Urol Esp 2005; 29 (2): 170-173

\section{RESUMEN}

INFLUENCIA DE LA INFLAMACIÓN CRÓNICA PROSTÁTICA EN MUESTRAS DE BIOPSIA SEXTANTE EN LOS NIVELES SÉRICOS DE PSA TOTAL Y PSA LIBRE

Objetivo: Analizar la influencia en los niveles séricos de PSA total (PSA) y PSA libre (PSA) de los signos de inflamación crónica detectados mediante biopsia prostática en pacientes no sintomáticos.

Pacientes y método: Seleccionamos 518 pacientes asintomáticos sometidos a ecografía transrectal y biopsia sextante de próstata por $\mathrm{PSA}_{\mathrm{t}}>4 \mathrm{ng} / \mathrm{mL}$ y/o tacto rectal sugestivo de malignidad. Seleccionamos aquellos pacientes en los que la biopsia descartaba malignidad. Se definieron dos grupos, pacientes con lesión benigna de próstata (LBP) y pacientes con infiltrado inflamatorio crónico (SIC).

Resultados: En 456 pacientes $(88,03 \%)$ la biopsia se realizó por elevación de PSA $_{t}$ o/y por tacto rectal sospechoso en 62 pacientes (11,97\%). El volumen medio prostático en pacientes con LBP fue $54 \mathrm{cc}$, siendo $51 \mathrm{cc}$ el volumen medio en pacientes con criterios histológicos de SIC. El PSA medio en $_{\mathrm{t}}$ pacientes con LBP fue 9,43 (IC 95\% $\pm 7,8$ ) y 8,8 (IC 95\% $\pm 5,73$ ) en el grupo de SIC.

Conclusiones: La presencia de SIC, no tiene influencia significativa en los valores PSA $_{1}$ y PSA en la $_{t}$ población estudiada. Estos hallazgos, a efectos de práctica clínica, permite en nuestro medio valorar las cifras de PSA con independencia de la presencia de SIC en las muestras de biopsia.

Palabras clave: Inflamación crónica. PSA. Biopsia de próstata.

\section{ABSTRACT}

ROLE OF CHRONIC PROSTATIC INFILTRATE FROM SPECIMENS OF PROSTATE BIOPSY IN TOTAL AND FREE PSA DETERMINATION

Purpose: To analyze the influence in total serum PSA (PSA) and free PSA (PSA) of chronic inflammatory patterns from prostate biopsy specimens of non-symptomatic patients.

Patients and methods: 518 non-symptomatic patients underwent ultrasonography and prostatic biopsy for $\mathrm{PSA}_{t}>4 \mathrm{ng} / \mathrm{mL}$ and/or DRE suspicious of malignancy. Those with a negative biopsy were divided into two subgroups: patients with benign prostatic lesions (LBP) and patients with chronic inflammatory signs (SIC).

Results: 456 patients (88.03\%) were biopsied for elevated $\mathrm{PSA}_{\mathrm{t}}$ or/and DRE suspicious of malignancy in 62 patients (11.97\%). Mean volume in patients with LBP was 54cc, while 51cc was the mean volume in patients with histological pattern of chronic inflammatory infiltrate. Mean $\mathrm{PSA}_{t}$ in patients with LBP was 9.43 (IC 95\% \pm 7.8 ) and 8.8 (IC 95\% \pm 5.73 ) in SIC's group patients.

Conclusions: The presence of SIC has no significant influence in the eventual value of PSA $_{1}$ y PSA in a selected population. As a result of that finding data from serum PSA can be reliably evaluated even in the presence of SIC in biopsy specimens.

Keywords: Chronic inflammation. PSA. Prostate biopsy. 
$\mathrm{E}^{1}$ antígeno prostático específico (PSA) es un marcador sérico utilizado en el screening y diagnóstico del cáncer de próstata así como en la monitorización de pacientes con HBP y cáncer de próstata $^{1-3}$. Sin embargo, éste es un marcador órgano-específico, con alta sensibilidad, pero baja especificidad para la detección del cáncer de próstata, existiendo situaciones que provocan una elevación temporal de los niveles de PSA, como son las manipulaciones de la glándula prostática (tacto rectal, ultrasonografía transrectal y biopsia, entre otras) ${ }^{4}$. Igualmente, patologías no tumorales se han relacionado con elevaciones sérica de los valores de $\mathrm{PSA}^{5,6}$, aunque su contribución en las cifras totales de PSA no están bien caracterizadas, como es el caso de la HBP e inflamación de la glándula prostática ${ }^{4,7}$. Estas patologías benignas que pueden elevar las cifras de PSA, son responsables de falsos positivos en la detección de cáncer de próstata basada en la determinación de los niveles de esta enzima según algunos estu$\operatorname{dios}^{8}$. Bajo esta condición, se realizan biopsias de próstata innecesarias, $\mathrm{y}$ en ocasiones pueden mostrar la presencia de patrones de inflamación de la glándula pese a no asociarse a sintomatología sugerente de prostatitis ${ }^{9}$.

El objetivo de este estudio ha sido analizar la influencia en los niveles séricos de PSA total $\left(\mathrm{PSA}_{t}\right)$ y porcentaje de PSA libre (PSA) de los signos de inflamación crónica detectados en la biopsia prostática sextante en pacientes no sintomáticos.

\section{PACIENTES Y MÉTODO}

Hemos realizado un estudio retrospectivo entre febrero de 1996 y diciembre de 2003, incluyendo a 518 pacientes asintomáticos, con una mediana de edad de 70 años, que habían sido sometidos a ecografía transrectal y biopsia sextante de próstata por cifras de $\mathrm{PSA}_{\mathrm{t}}>4$ $\mathrm{ng} / \mathrm{mL}$ y/o tacto rectal sugestivo de malignidades no evidenciada en la biopsia.

La detección de $\mathrm{PSA}_{t}$ se realizó con el reactivo comercial Inmulyte PSA ${ }^{\circledR}$ o por técnica de hibridación con el reactivo Hybritech-Tandem- ${ }^{\circledR}$. La determinación del $\mathrm{PSA}_{1}$ se realizó con el reactivo Inmulyte PSA ${ }^{\circledR}$. En todos los pacientes se calculó el volumen prostático mediante ultrasonografía transrectal.
Seleccionamos aquellos pacientes en los que la biopsia descartaba signos de malignidad, definiendo dos grupos en función de los resultados histopatológicos en las muestras de biopsia obtenidas ${ }^{10}$ :

- Signos inflamatorios crónicos (SIC), que se definió por un infiltrado celular de predominio linfocitario y plasmocelular, que condiciona cambios epiteliales de regeneración, con variaciones nucleares y distribución cribosa. En ocasiones, el infiltrado crónico se mezcla con algún granuloma o la presencia de histiocitos espumosos.

- Lesión benigna de próstata (LBP), que se definió como el incremento de todos los elementos histológicos de la próstata; epitelio hiperplásico a partir de las glándulas preexistentes, y un incremento en la densidad de los tejidos estromales (tejido conectivo y músculo liso), con crecimiento de características nodulares con ambos componentes en proporciones variables (adenomiofibromatoso, fibromuscular, muscular puro, fibroadenomatoso y estromal puro).

Análisis estadístico: Como test estadístico se utilizó la T de Student para comparar los valores medios de las variables a estudio; volumen, $\mathrm{PSA}_{t}$ y PSA.

\section{RESULTADOS}

De los 518 pacientes, en 456 pacientes $(88,03 \%)$ la biopsia se realizó por elevación de las cifras de $\mathrm{PSA}_{\mathrm{t}} \mathrm{o} / \mathrm{y}$ por tacto rectal sospechoso en 62 pacientes (11,97\%). En la mayoría de los pacientes (445 pacientes) era su primera biopsia (85,9\%), en 61 pacientes era la segunda biopsia $(11,78 \%)$ y en el $2,32 \%$ se trataba de la tercera biopsia prostática. El análisis histológico de la pieza evidenció LBP en 433 pacientes $(83,65 \%)$ y SIC en 85 pacientes (16,35\%).

Para cada grupo definido en función de los hallazgos histopatológicos calculamos y comparamos la media del volumen prostático, media de $\mathrm{PSA}_{\mathrm{T}}$ y media del PSA . El volumen medio prostático en el grupo de pacientes con LBP fue de $54 \mathrm{cc}$, siendo de $51 \mathrm{cc}$ el volumen medio en pacientes con criterios histológicos de SIC. En cuanto a los valores de PSA, el $\mathrm{PSA}_{\mathrm{t}}$ medio en los pacientes con LBP fue de 9,43 (IC 95\% $\pm 7,8$ ) y de 8,8 (IC $95 \% \pm 5,73$ ) en el grupo de SIC. El PSA medio en 
los pacientes con LBP fue de 21 (IC 95\% $\pm 6,8$ ) frente a 16 (IC 95\% $\pm 8,7$ ) en el grupo de pacientes con criterios histológicos de SIC.

Al comparar los resultados de ambos grupos, mediante el test estadístico T de Student para la comparación de medias, no encontramos diferencias estadísticamente significativas en ninguna de las tres variables estudiadas. Ambos grupos tienen valores de volúmenes prostáticos similares

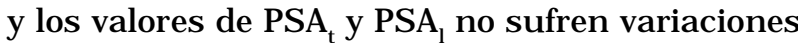
por la presencia de signos inflamatorios en la biopsia prostática al compararlo con los pacientes sin inflamación y con LBP, lo que demuestra que su presencia no es un factor que modifique dichos valores (Tabla 1 ).

\section{Tabla 1}

Comparación de las medias de las variables volumen, $\mathrm{PSA}_{\mathrm{t}}$ y $\mathrm{PSA}_{1}$, entre pacientes con LBP e IC.

\begin{tabular}{lcccc}
\hline & número & volumen & PSA $_{\mathbf{t}}$ & PSA $_{1}$ \\
\hline LBP & 433 & 54 & $9,43 \pm 7,8$ & $21 \pm 6,8$ \\
SIC & 85 & 51 & $8,8 \pm 5,73$ & $16 \pm 8,7$ \\
valor de p & & $0,45^{*}$ & $0,5^{*}$ & $0,48^{*}$ \\
\hline
\end{tabular}

* T de Student

(SIC: infiltrado crónico inflamatorio. LBP: lesión benigna de próstata)

\section{DISCUSIÓN}

La influencia de la inflamación crónica de la glándula prostática en los valores séricos de PSA, ha sido estudiada con resultados controvertidos publicados en la literatura, probablemente debidos a la heterogenicidad en el diseño de los distintos estudios.

Distintas publicaciones revelan una gran variabilidad en la prevalencia de signos inflamatorios crónicos prostáticos, que oscilan entre un $5 \%$ y un $98 \%$, diagnosticados mediante el análisis de muestras anatomo-patológicas procedentes de biopsia con aguja, autopsias y resecciones transuretrales ${ }^{11,12}$.

Sin embargo, en el presente estudio, obtenemos un bajo porcentaje de pacientes $(16,35 \%)$ con resultado anatomopatológico de SIC cuando lo comparamos con otros estudios publicados. Así, Bozeman et al. ${ }^{5}$, en un estudio retrospectivo sobre 137 pacientes, encuentra una prevalencia de SIC del $72,5 \%$ en las biopsias realizadas por sospecha de cáncer de próstata. Nadler et al. ${ }^{12}$ describen que el 98,6\% de los pacientes con PSA elevado y el $77,4 \%$ de los pacientes con PSA normal sometidos a biopsia de próstata, tenían criterios histológicos de SIC. Esta gran variabilidad es probablemente debida a distintos factores, como la interpretación de los signos inflamatorios prostáticos por parte del patólogo, que debe ser cuidadosa, ya que en las muestras remitidas tras biopsia no es raro encontrar pequeños focos aislados de inflamación, reservando el diagnóstico para lesiones significativas. De la misma forma la localización de las muestras analizadas pueden variar la detección de los signos inflamatorios, dado que la inflamación se ubica predominantemente en la zona periférica, en menor medida en la zona transicional (sobretodo relacionado con procesos endocavitarios) y rara vez en la zona central. Por otra parte, las diferencias en la detección de signos inflamatorios en las distintas series también se justifican por la subjetividad del criterio anatomo-patológico utilizado, aunque en los patrones inflamatorios la morfología es el criterio predominante. Así pues, las diferencias de porcentaje de SIC en las muestras analizadas en el presente estudio, hay que buscarlas en los criterios anatomopatológicos que se han utilizado para clasificar a los pacientes, en la heterogenicidad del diseño de los estudios comparados (sólo biopsiamos pacientes con PSA > 4 y/o tacto rectal sugestivo) y en menor medida en las posibles diferencias poblacionales.

Existe una gran disparidad en los resultados descritos en la literatura. Entre los estudios más relevantes, encontramos que en la serie aportada por Bozeman sobre 51 pacientes sometidos a biopsia prostática de 95 totales, en 37 de ellos evidencia un patrón inflamatorio que califica como prostatitis crónica y lo relaciona como una causa de incremento en cifras de PSA, sin embargo no valora el tamaño prostático ${ }^{13}$ a la hora de analizar los resultados y por otro lado, estudia conjuntamente los pacientes con diagnóstico anatomopatológico de prostatitis crónica con los diagnosticados mediante análisis de secreción prostática. Brawn et al. ${ }^{5}$ analizan los resultados de 105 autopsias de varones que en el año previo al éxitus se habían sometido a tato rectal y análisis de PSA y encuentran que la pro- 
porción de pacientes con PSA $>4 \mathrm{ng} / \mathrm{ml}$ era estadísticamente superior en el grupo con infiltrado prostático agudo o crónico que en el resto de pacientes. Sin embargo 37 de los 105 pacientes tenían criterios histológicos de cáncer de próstata, no se trataba de pacientes obtenidos mediante screening o diagnóstico precoz poblacional y algunos de los pacientes eran portadores de catéteres uretrales, por lo que no era una muestra homogénea y por tanto no era posible extrapolar los resultados a otras poblaciones. Nadler ${ }^{12}$ demuestra cuantitativamente que la inflamación aguda y crónica, en ausencia de prostatitis clínica, se asocia a un ligero incremento en las cifras de PSA en el estudio univariante, aunque su análisis de regresión evidencia que la variable que más contribuye al incremento de las cifras de PSA, es el volumen prostático.

Por el contrario, el estudio de Morote et al. ${ }^{14}$ no revela una influencia significativa el hallazgo de infiltrado inflamatorio crónico en los valores de PSA en su serie de 284 pacientes. En esta misma línea, nuestra serie con 518 pacientes demuestra que la presencia de SIC en muestras procedentes de biopsias sin hallazgos histológicos de cáncer, no tiene influencia significativa en los valores finales de $\mathrm{PSA}_{1}$ y $\mathrm{PSA}_{t}$ en la población estudiada. Con estos hallazgos y a efectos de práctica clínica, nos permite en nuestro medio, valorar las cifras de PSA con independencia de la presencia de SIC en las muestras de biopsia.

\section{REFERENCIAS}

1. Stamey TA, Yang N, Hay AR, Mcneal JE, Freiha FS, Redwine E. Prostate-specific antigen as a serum marker for adenocarcinoma of the prostate. N Engl J Med 1987;317:909-916.

2. Hudson MA, Bahnson RR, Catalona WJ. Clinical use of prostate specific antigen in patients with prostate cancer. J Urol 1989;142:1011-1017.

3. Catalona WJ, Smith DS, Ratliff TL, Dodds KM, Coplen DE, Yuan JJ, et al. Measurement of prostate-specific antigen in serum as a screening test for prostate cancer. N Engl J Med 1991; 324:1156-1161.
4. Polascik TJ Oesterling JE, Partin AW. Prostate specific antigen: a decade of discovery-what we have learned and where we are going. J Urol 1999;162:293-306.

5. Bozeman CB, Carver BS, Eastham JA, Venable DD. Treatment of chronic prostatitis lowers serum prostate specific antigen. J Urol 2002;167:1723-1726.

6. Brawn PN, Speights VO, Kuhl D, Riggs M, Spiekerman AM, Mccord RG, et al. Prostate-specific antigen levels from completely sectioned, clinically benign, whole prostates. Cancer 1991;68:1592-1599.

7. Soler Soler JL, Martínez Torres JL, Hidalgo Domínguez MR, Lardelli Claret P, Liebana Urena J, Zuluaga Gómez A, et al. Inflammatory changes in the obstructed prostate: the correlation between the bacteriological and histological findings. Arch Esp Urol 1999;52:729-738.

8. Ornstein DK, Smith DS, Humphrey PA, Catalona WJ. The effect of prostate volume, age, total prostate specific antigen level and acute inflammation on the percentage of free serum prostate specific antigen levels in men without clinically detectable prostate cancer. J Urol 1998;159: 1234-1237.

9. Jung K, Meyer A, Lein M, Rudolph B, Schnorr D, Loenig SA. Ratio of free-to-total prostate specific antigen in serum cannot distinguish patients with prostate cancer from those with chronic inflammation of the prostate. J Urol 1998 May; 159 (5):1595-1598.

10. Kohnen PW, Drach GW. Patterns of inflammation in prostatic hyperplasia: a histologic and bacteriologic study. J Urol 1979;121:755-760.

11. Irani J, Levillain P, Goujon JM, Bon D, Dore B, Aubert J. Inflammation in benign prostatic hyperplasia: correlation with prostate specific antigen value. J Urol, 1997;157(4): 1301-1303.

12. Nadler RB, Humphrey PA, Smith DS, Catalona WJ, Ratliff TL. Effect of inflammation and benign prostatic hyperplasia on elevated serum prostate specific antigen levels. $J$ Urol, 1995;154(2 Pt 1): 407-413.

13. Hasui Y, Marutsuka K, Asada Y, Ide H, Nishi S, Osada Y. Relationship between serum prostate specific antigen and histological prostatitis in patients with benign prostatic hyperplasia. Prostate 1994;25(2):91-96.

14. Morote J, López M, Encabo G, De Torres IM. Effect of inflammation and benign prostatic enlargement on total and percent free serum prostatic specific antigen. Eur Urol 2000;37:537-540.

Dr. L. Gómez Pérez.

Secretaría de Urología. Pabellón Central.

Hospital Universitario La Fe

Av. Campanar no 9 - 46021 Valencia

(Trabajo recibido el 22 octubre de 2004) 